\title{
Ectopic Spleen Tissue - an Underestimated Differential Diagnosis of a Hypervascularised Liver Tumour
}

\author{
Eberhard Grambow ${ }^{a} \quad$ Malte Weinrich ${ }^{a} \quad$ Annette Zimpfer ${ }^{b}$ Katja Kloker ${ }^{c}$ Ernst Klar ${ }^{a}$ \\ a Department of General, Thoracic, Vascular and Transplantation Surgery, University of Rostock, Rostock, Germany, \\ b Institute of Pathology, University of Rostock, Rostock, Germany, \\ c Institute of Diagnostic and Interventional Radiology, University of Rostock, Rostock, Germany
}

\section{Keywords}

Liver tumour - Hypervascularised tumour .

Liver resection - Splenosis

\section{Summary}

Background: Patients with liver cirrhosis have an increased risk of developing hepatocellular carcinoma (HCC). Implantation metastasis following diagnostic biopsy is a well-known complication. Therefore, primary resection of a hypervascularised tumour suspicious for HCC is often performed with curative intent. Case Report: An exophytically growing mass was diagnosed between liver segments III and IVb by means of ultrasound in a 53-year old male patient with decompensated liver cirrhosis. Computed tomography confirmed a $3.5 \mathrm{~cm}$ large hypervascularised tumour with given resectability. Intraoperatively, the tumour appeared like a HCC. Thus, an atypical resection was performed. Histopathology revealed ectopic spleen tissue without any signs of malignancy. As enquiries revealed, the patient had undergone splenectomy after a blunt abdominal trauma 9 years prior to admission. Conclusion: In the present patient, hepatic splenosis in a cirrhotic liver was misinterpreted as HCC. In patients with a history of traumatic rupture of the spleen or splenectomy, splenosis has to be considered as a potential differential diagnosis of a hypervascularised tumour. Specific diagnostics should be performed to rule out splenosis.

(c) 2015 S. Karger GmbH, Freiburg

\section{Introduction}

Hepatocellular carcinoma (HCC) is one of the most common carcinomas worldwide and is associated with a significant cancerrelated mortality [1]. In patients with liver cirrhosis the risk of developing HCC is markedly increased. Therefore, in these patients HCC screening is recommended by means of ultrasound (US) examination or measurement of alpha-fetoprotein $[2,3]$.

Tumours suspicious for HCC are further diagnosed by computed tomography (CT) or magnetic resonance imaging (MRI) to gain information about their localization and to evaluate given resectability. In order to assure the diagnosis, percutaneous liver biopsy might be considered. Thus, a HCC can be histologically differentiated from metastasis or other tumour entities [4]. Transcutaneous biopsies are often avoided due to the risk of bleeding and metastatic spread, which occurs in $0.0095-5 \%$ [5]. Therefore, primary liver resection is often performed in patients with an increased risk for bleeding complications and assumed HCC.

\section{Case Report}

A 53-year-old male patient was initially admitted to the Department of Gastroenterology, University of Rostock, Germany, due to decompensated ChildPugh class B liver cirrhosis based on a history of severe alcohol abuse. The patient suffered from therapy-refractory ascites, multiple gastric ulcers, duodenitis, sigma diverticulosis, and a condition after hepatorenal syndrome. Regarding the past medical history, the patient reported of a splenectomy after an industrial accident with traumatic rupture of the spleen and left-sided hematothorax 9 years ago. Routine US revealed a space-occupying mass of approximately 3.5 $\mathrm{cm}$ size in the central part of the liver (fig. 1). As a second, independent imaging modality CT revealed a contrast enhancement typical for HCC within the tumour (fig. 2). The plasmatic level of alfa-fetoprotein was within normal range.

After presentation of the case and synopsis of the actual findings in the in terdisciplinary tumour board, primary resection of the tumour was recommended. The patient was transferred to our department and underwent explorative laparotomy. Intraoperatively, moderate adhesions between organs and peritoneum were found. The liver showed a fine nodular sclerosis with the exo-

\section{KARGER \\ Fax +497614520714


Fig. 1. a Native and $\mathbf{b}$ contrast-enhanced US scan showing a hypervascularized hepatic tumour.
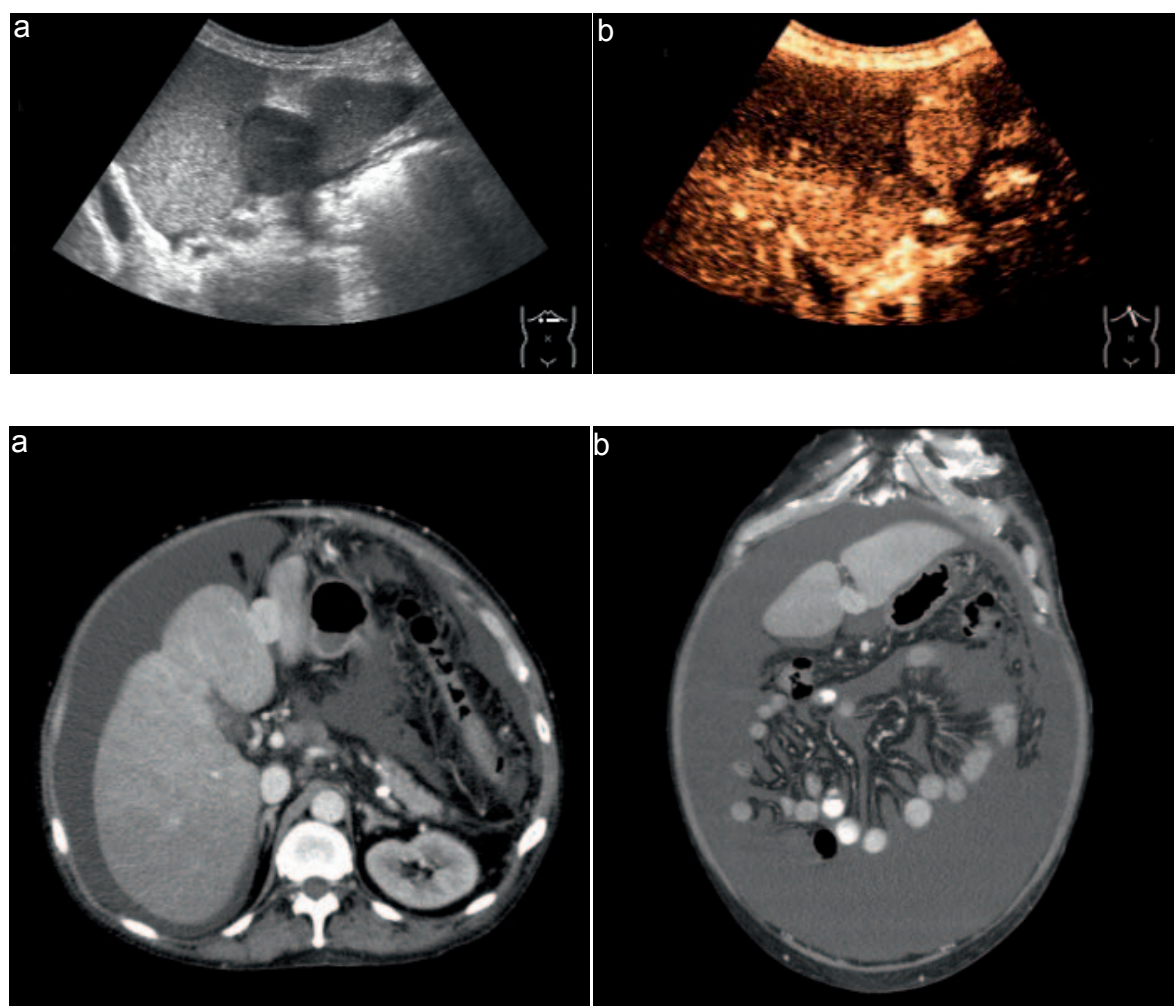

Fig. 2. a Axial and $\mathbf{b}$ coronal contrast-enhanced CT scan showing a hypervascularized mass between liver segments III and IVb.
In the present case, the clinical presentation of the patient with liver cirrhosis and a hypervascularised hepatic mass raised our suspicion of a HCC, not considering the differential diagnosis of a splenosis with the background of splenectomy due to traumatic rupture of the spleen. In addition to our case, other rare case reports show that splenosis is still often misdiagnosed as a malignant tumour [8-10], leading to unnecessary major surgery. Splenosis should be considered as a rare but important differential diagnosis of hypervascularised tumours, especially in patients with a past medical history of a traumatic rupture of the spleen or splenectomy $[11,12]$.

In order to rule out splenosis from the differential diagnosis preoperatively, histopathological examination based on tissue gained by needle aspiration prior to operation [13] or scintigraphy with $(99 \mathrm{~m}) \mathrm{Tc}$-labelled head-denatured erythrocytes, a technique with the greatest specificity in the demonstration of splenic tissue, should be performed [14]. However, the risk of tumour dissemination following needle biopsy has to be considered [5]. A further diagnostic option is MRI with gadoxetic acid. Due to this liver-specific contrast agent both sensitivity and specificity for detecting and characterising liver lesions by means of MRI are markedly increased [15]. However, similarly to the surgeon, the radiologist has to be aware of a possible splenosis during assessment of the images. Finally, rapid pathological examination might be required intraoperatively if a splenosis is potentially reasonable due to the patient's past medical history of a traumatic rupture of the spleen or a splenectomy. 
Fig. 3. a Low-power view of an exophytic mass adherent to the liver (H\&E; original magnification $\times 5)$; b high-power view of the mass demonstrating splenic tissue with hemosiderosis (H\&E; original magnification $\times 100$ ).
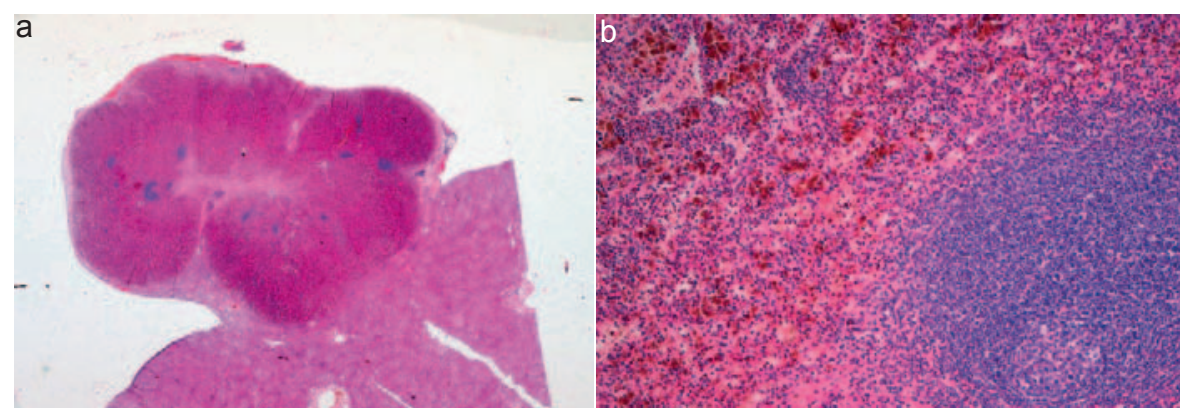

\section{Conclusion}

Knowledge of the possibility and consideration of a splenosis is crucial to avoid unnecessary surgery in patients with hypervascularised tumours of unknown aetiology and a past medical history of traumatic rupture of the spleen. This equally counts for patients after splenectomy due to other reasons. Even though another diagnosis seems to be more likely, it is quite important to perform further diagnostics, such as scintigraphy or contrast-enhanced MRI, prior to resection or ablation especially in patients with hypervascularised tumours of the liver close to the surface with a high risk for bleeding complications during diagnostic biopsy.

\section{Disclosure Statement}

The authors report no declarations of interest.

\section{References}

1 Bosch FX, Ribes J, Díaz M, Cléries R: Primary liver cancer: worldwide incidence and trends. Gastroenterology 2004;127:S5-S16.

2 Sherman M: The radiological diagnosis of hepatocellular carcinoma. Am J Gastroenterol 2010;105:610-612.

> Singal A, Volk ML, Waljee A, Salgia R, Higgins P, Rogers MA, Marrero JA: Meta-analysis: surveillance with ultrasound for early-stage hepatocellular carcinoma in patients with cirrhosis. Aliment Pharmacol Ther 2009; 30:37-47.

4 Tannapfel A, Dienes HP, Lohse AW: The indications for liver biopsy. Dtsch Aerztebl Int 2012;109:477-483.

$\checkmark 5$ Silva MA, Hegab B, Hyde C, Guo B, Buckels JA, Mirza DF: Needle track seeding following biopsy of liver lesions in the diagnosis of hepatocellular cancer: a systematic review and meta-analysis. Gut 2008;57:15921596.

6 Buchbinder JH, Lipkoff CJ: Splenosis: multiple peritoneal splenic implant following abdominal injury. Surgery 1939;6:927-934.
7 Fleming CR, Dickson ER, Harrison EG: Splenosis: autotransplantation of splenic tissue. Am J Med 1976;61: 414-419.

8 Kang KC, Cho GS, Chung GA, Kang GH, Kim YJ, Lee MS, Kim HK, Park SJ: Intrahepatic splenosis mimicking liver metastasis in a patient with gastric cancer. J Gastric Cancer 2011;11:64-68.

-9 Liu Y, Ji B, Wang G, Wang Y: Abdominal multiple splenosis mimicking liver and colon tumors: a case report and review of the literature. Int J Med Sci 2012;9: 174-177.

10 Kandil TS, El Sorogy M, Naiem Y, Elkashef WF: Postsplenectomy splenosis presenting as hepatocellular carcinoma in the left lateral section of the liver: a case report. Int J Surg Case Rep 2014;5:877-878.

11 Yeh CJ, Chuang WY, Kuo TT: Unusual subcutaneous splenosis occurring in a gunshot wound scar: pathology and immunohistochemical identification. Pathol Int 2006;56:336-339.
12 Khan AM, Manzoor K, Gordon D, Berman A: Thoracic splenosis: a diagnosis by history and imaging. Respirology 2008;13:481-483.

13 Renne G, Coci A, Biraghi T, Schmid C: Fine needle aspiration of thoracic splenosis. A case report. Acta Cytol 1999;43:492-494.

14 Menth M, Herrmann K, Haug A, Raziorrouh B, Zachoval R, Jung CM, Otto C: Intra-hepatic splenosis as an unexpected cause of a focal liver lesion in a patient with hepatitis $\mathrm{C}$ and liver cirrhosis: a case report. Cases J 2009;2:8335.

15 Jhaveri K, Cleary S, Audet P, Balaa F, Bhayana D, Burak K, Chang S, Dixon E, Haider M, Molinari M, Reinhold C, Sherman M: Consensus statements from a multidisciplinary expert panel on the utilization and application of a liver-specific MRI contrast agent (gadoxetic acid). AJR Am J Roentgenol 2015;204:498509. 